\title{
Über theoretische und reale Recyclingfähigkeit
}

\author{
Roland Pomberger
}

Online publiziert: 29. Oktober 2020

(C) Der/die Autor(en) 2020

Zusammenfassung Zukünftig sollte die Recyclingfähigkeit neu definiert werden. Erkennbarkeit und Sortierfähigkeit, aber auch die Eignung der Sammelsysteme sind unbedingt zu berücksichtigen. Die Prüfung der Recyclingfähigkeit soll auch durch experimentelle Tests abgesichert werden. In diesem Artikel werden das Stufenmodell der Recyclingfähigkeit und die Methode der Material-Nutzungszeit vorgestellt.

Wenn wir zukünftig mehr und besser recyclingfähige Produkte in unseren abfallwirtschaftlichen Anlagen finden, werden wir technisch auch in der Lage sein, diese $\mathrm{zu}$ separieren und $\mathrm{zu}$ verwerten. Der Gesetzgeber ist aufgerufen, für diese Privilegierung von recyclingfähigen Produkten die geeigneten Rahmenbedingungen und Anreizsysteme $\mathrm{zu}$ schaffen. Ökomodulation ist dazu ein wirksames Mittel.

Schlüsselwörter Theoretische, technische und reale

Recyclingfähigkeit - Material-

Nutzungszeit · Recyclingquoten

\section{About theoretical and real recyclability}

Abstract In future, recyclability should be redefined. Recognizability and sortability, but also the suitability of the collection systems must be taken into account. The recyclability should also be verified by experimental tests. In this article, the step-by-step model of recyclability and the method of material utilization time are presented. If we find more and better recyclable products in our waste management plants in the future, we will be technically able to separate and recycle them. The legislator is called upon to create the

Univ.-Prof. DI Dr. R. Pomberger ( $\square)$ Lehrstuhl für

Abfallverwertungstechnik

und Abfallwirtschaft,

Montanuniversität Leoben,

Franz-Josef-Straße 18, 8700 Leoben,

Österreich

avaw@unileoben.ac.at appropriate framework and incentive systems for this privileged treatment of recyclable products. Eco-modulation is an effective way of doing this.

Keywords Theoretical, technical and real recyclability - Material usage time . Recycling rates

\section{Einleitung}

Immer mehr Produkte werben mit den Attributen „recyclingfähig“ oder „kompostierfähig“ und signalisieren den Konsumenten damit besondere Umweltverträglichkeit. Aus Sicht der Abfallwirtschaft ist die Frage berechtigt, ob diese Produkte wirklich recycelt werden und wie sie sich in unserem REALEN abfallwirtschaftlichen System verhalten.

Bei genauerem Hinsehen lösen sich leider viele Versprechungen in Luft auf. Die Recyclingfähigkeit bezieht sich oft nur auf den Werkstoff, der theoretisch stofflich verwertet werden könnte. Damit ist aber die technische Recyclingfähigkeit noch lange nicht gegeben. Dazu gehört nämlich auch noch die Erkennbarkeit z.B. durch Sensoren, aber auch die Ausschleusbarkeit in Sortiermaschinen, um ein Partikel aus einem Gutstrom abzuscheiden. Zudem muss auch ein geeignetes Recyclingverfahren für das in der Sortierung gewonnene Konzentrat vorhanden und nutzbar sein.

In realen abfallwirtschaften Systemen sind aber zusätzlich noch das Verhalten des Abfallerzeugers, das Vorhandensein und der Einfluss des Sammelsystems, die reale Verschmutzung und die regionalen Aspekte, wie die Existenz von geeigneten Sortieranlagen, heranzuziehen. Wenn all das berücksichtigt wird, dann können wir von der realen Recyclingfähigkeit sprechen.

\section{Rahmenbedingungen}

Derzeit wird die Diskussion zum Thema Recyclingfähigkeit weitgehend auf Verpackungen und hier im Speziellen auf Kunststoffverpackungen reduziert.
Kunststoffverpackungsabfälle stellen aber nur einen relativ kleinen Abfallstrom dar, auf Österreich bezogen nur etwa $3,7 \%$ der Siedlungsabfälle oder $0,25 \%$ aller Abfälle. Allerdings zeigt sich, dass gerade Produkte aus Kunststoff und hier besonders die Kunststoffverpackungen, noch nicht jene Recyclingquoten erreichen konnten wie Produkte aus anderen Werkstoffen. Es ist evident, dass der Werkstoff Kunststoff in Hinblick auf seine Kreislauffähigkeit noch deutliches Nachholpotenzial aufweist.

Die Reduktion der Diskussion auf Kunststoffprodukte greift allerdings $\mathrm{zu}$ kurz. Wenn wir mehr rezyklieren wollen, müssen wir alle Produkte, die zu Siedlungsabfall werden, in ihrer Recyclingfähigkeit verbessern. Die Forderung nach verbesserter Recyclingfähigkeit ist grundsätzlich an alle Produkte $\mathrm{zu}$ stellen, allerdings gelten gesetzliche Vorgaben an die Recyclingfähigkeit, ausgedrückt durch Sammel- und Verwertungsquoten, nur für bestimmte Produkte bzw. deren Abfallströme.

Aufgrund der aktuellen Diskussion und der offensichtlichen Notwendigkeit von besserer Recyclingfähigkeit von Kunststoffprodukten bzw. deren Abfällen wird im Folgenden die Recyclingfähigkeit von Kunststoffabfällen besondere Aufmerksamkeit gewidmet.

\subsection{Ausgangslage für Kunststoffabfälle - Neue Europäische \\ Rahmenbedingungen}

Mit der Kunststoffstrategie der Europäischen Kommission (2018) will die Europäische Union eine Grundlage für eine neue Bewirtschaftung von Kunststoffmaterialien schaffen. Bei der Gestaltung und Herstellung von Kunststoffprodukten soll den Erfordernissen in Bezug auf Vermeidung, Vorbereitung zur Wiederverwendung, Reparatur und Recycling in vollem Umfang Rechnung getragen werden. $\mathrm{Zu}$ den Hauptzielen gehören die Erhöhung der Wertschöpfung und die Verminderung der Verschmutzung sowie der schädlichen Auswirkungen auf Mensch und Umwelt durch Kunststoffe. Zur Erreichung dieser Ziele sind 
ausdrücklich auch zukünftige Produkte adressiert, die vermehrt stofflich verwertbar (recyclingfähig) gestaltet werden sollen.

Um dem Zukunftsbild einer Kreislaufwirtschaft näher zu kommen, sind in der Kunststoffstrategie Maßnahmen aufgelistet, welche auch auf nationaler und regionaler Ebene umgesetzt werden sollten. Darunter ist insbesondere die Forderung nach recyclingfreundlicher Gestaltung von Produkten gemeint, damit diese am Ende ihres Lebenszyklus (EoL) verstärkt dem Recycling zugeführt werden können.

Das Ziel der EU-Kunststoffstrategie, bis $2030100 \%$ wiederverwendbare oder recyclingfähige Kunststoffe (nicht nur Verpackungen) zu erreichen, ist sehr ambitioniert. Das wird auch durch unternommene Potenzialbetrachtungen deutlich.

\subsection{Recyclingquoten}

Die Erhöhung der Recyclingquote stellt dabei ein maßgebliches Instrument der Europäischen Union und deren Mitgliedstaaten dar, um insbesondere
Treibhausgaseinsparungen $\mathrm{zu}$ erreichen. Die neuen EU-weiten Rahmenbedingungen basieren maßgeblich auf dem EU-Kreislaufwirtschaftspaket und auf der EU Plastics Strategy, die sich auch in entsprechenden Aktionsplänen und nicht zuletzt im Abfallwirtschaftsgesetz niederschlagen. Neben daraus hervorgegangenen strengen Vorgaben wie der Single-Use Plastics Directive (SUP-Richtlinie) (Europäisches Parlament 2019) und der Abfallrahmenrichtlinie werden in Studien und Strategien die Erhöhung des Recyclings und die Verbesserung der Kreislaufwirtschaft zu diesem Zweck maßgeblich gefordert. Hierzu sind ein stärkerer Produktfokus sowie die Erweiterung der Herstellerverantwortung essenziell. Nur unter Einbeziehung der Hersteller von Produkten ist die Einhaltung der gestiegenen Kunststoffrecyclingquoten unter massiven Anstrengungen möglich.

\subsection{Spezielle Recyclingquoten für Verpackungen}

In der EU-Verpackungsrichtlinie 1994 wurden mit der Novelle 2018 den Mit- gliedstaaten neue und höhere Recyclingraten für Verpackungen auferlegt. Bis zum Jahr 2030 sollen mindestens $70 \%$ aller in Verkehr gebrachten Verpackungen recycelt werden. Für Kunststoffverpackungen liegt das Mindestziel für 2025 bei $50 \%$ und für 2030 bei $55 \%$. Bisher lag das Ziel für 2025 bei 22,5\%.

Im Rahmen der erweiterten Herstellerverantwortung sollen weniger Verpackungsabfälle entstehen, diese dafür vermehrt getrennt gesammelt und recycelt werden. Diesbezüglich liegt es in der Verantwortung des Herstellers, seine Verpackungen selbst oder durch ein genehmigtes Sammel- und Verwertungssystem einer geeigneten Verwertung zuzuführen.

Neben der Quantität sind auch Qualitätsnormen von den Mitgliedstaaten $\mathrm{zu}$ erfüllen, um ein möglichst hochwertiges Recycling von Verpackungsabfällen und Verbundverpackungen $\mathrm{zu}$ ermöglichen. Die Mitgliedstaaten wurden aufgefordert, geeignete Maßnahmen zu treffen, um den Anteil an wiederverwendbaren Verpackungen zu steigern und gleichzeitig den Verbrauch

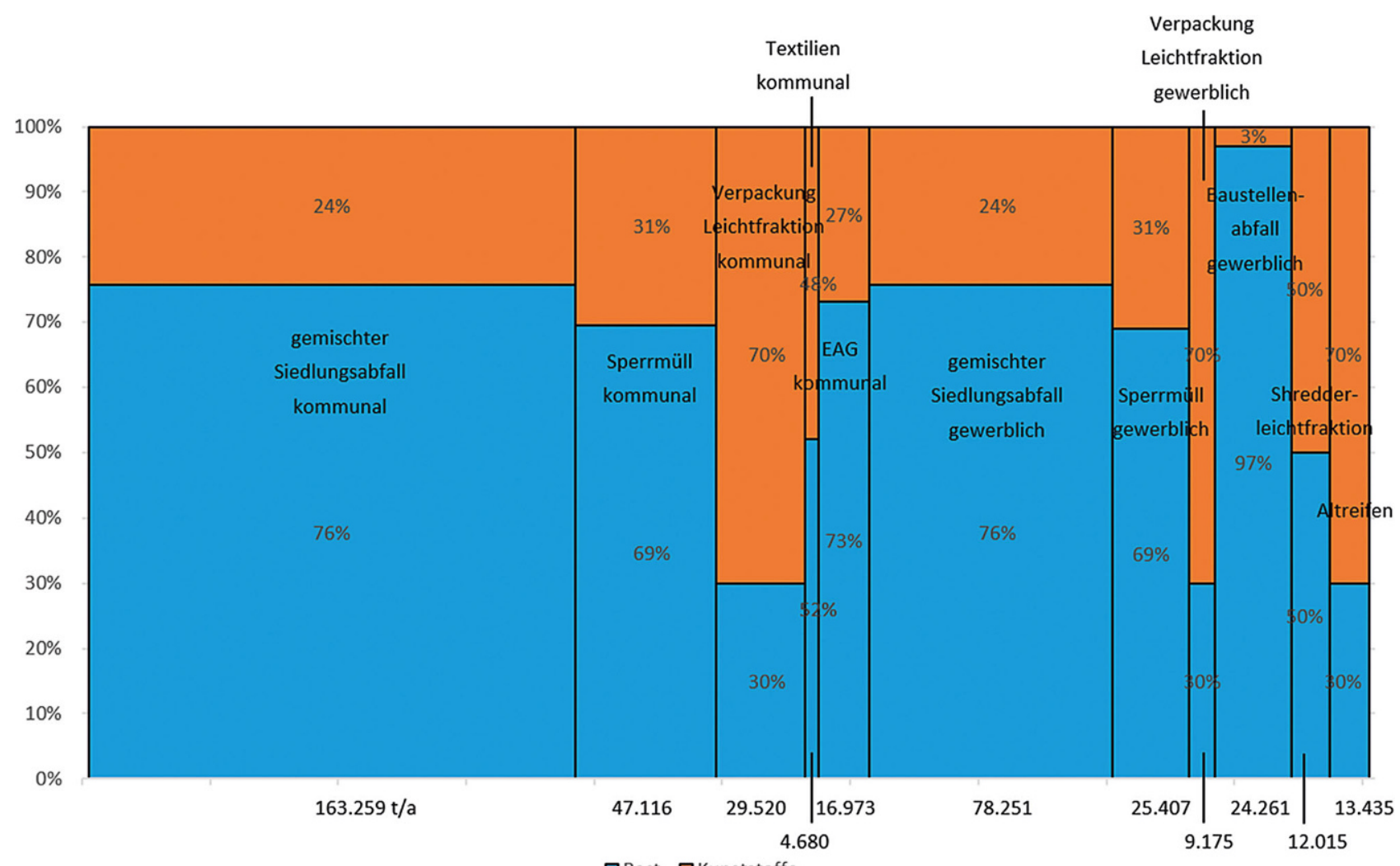

Abb. 1 Marimekko-Diagramm über die Kunststoff enthaltenden Abfallfraktionen in der Steiermark und deren Kunststoff-Anteil 2018. Diex-Achse zeigt die Menge der Abfallfraktionen in t/a, die y-Achse das Verhältnis zwischen dem Kunststoffanteil (orange) und dem Rest (blau) innerhalb einer Abfallfraktion (Wellacher et al. 2020) 
von nicht recycelbaren Verpackungen zu minimieren.

\subsection{Fallbeispiel Steiermark}

In der Steiermark fallen im Jahr etwa 425.000 t/a (2018) kunststoffhaltige Abfälle an (Abb. 1). Kunststoffe machen davon etwa 130.000 t/a aus, wobei insgesamt lediglich ca. $800 \mathrm{t} / \mathrm{a}$ wiederverwendet und etwa 12.000 t/a zu Kunststoffrezyklaten verarbeitet werden können. Damit liegt die aktuelle Recyclingquote bei unter $10 \%$ (bei Leichtverpackungen, mit $70 \%$ Kunststoffanteil, beträgt die Recyclingquote ca. $25 \%$ ) (Wellacher et al. 2020).

\section{Problemfelder}

Beispielhaft werden folgende Problemfelder angeführt.

\subsection{Wieviel wird wirklich im Kreislauf geführt?}

Die Recyclingrate ist eine ungenügende Kennzahl, um zu bewerten, wieviel Rohstoffe eines Produktes tatsächlich stofflich verwertet werden.

Es wird daher der Begriff „Kreis-

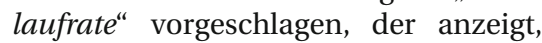
wieviel von einem gleichwertigen Vorläuferprodukt wieder in dasselbe Produkt rezykliert wurde. Die Kreislaufrate ergibt sich aus dem Produkt von Sammelquote, Sortierquote, Recyclingquote und Rezyklateinsatzquote. Ein Einwegprodukt mit $80 \%$ Sammelquote, $80 \%$ Sortierquote, $80 \%$ Recyclingquote und $80 \%$ Rezyklateinsatzquote ins selbe Produkt hätte somit eine Kreislaufquote von $41 \%$ (vgl. auch Abb. 9).

Beispielhaft ist hier die Vorschrift der EU-Single-Use-Plastics-Richtlinie $2019 \mathrm{zu}$ sehen, die für PET-KunststoffEinweg-Getränkeflaschen einen Rezyklatanteil von $25 \%$ bis 2025 und $30 \%$ bis 2030 vorschreibt. Das wird in Österreich nur gelingen, wenn ausreichend Postconsumer-PET importiert wird. Für allein in Österreich gesammeltes PostConsumer-PET liegt der Rezyklateinsatz in Österreich konsumierten PETFlaschen bei gerade einmal $14 \%$ (Wellacher et al. 2020).

\subsection{Greenwashing}

Als "greenwashing" werden Kampagnen und PR-Aktionen bezeichnet, die einzelne Produkte, ganze Unternehmen oder politische Strategien in ein „grü- nes“ Licht stellen, sodass der Eindruck entsteht, die Akteure würden besonders umweltfreundlich, ethisch korrekt und fair handeln. Unternehmen, die Greenwashing betreiben, treten in den Augen der Käufer und der Öffentlichkeit mit einem grünen Image auf und verkaufen dem Konsumenten das Prädikat „ökologisch wertvoll“. Bei den grüngewaschenen Produkten entspricht dieser äußere Schein allerdings nicht den ökologischen Tatsachen.

Um erfolgreichere Öffentlichkeitsarbeit betreiben zu können sowie den Marken- und Unternehmenswert zu steigern, missbrauchen die Unternehmen dabei die Grundideen der Corporate Social Responsibility (CSR), die unter dem Motto „Tue Gutes und sprich darüber"stehen und nachhaltiges Wirtschaften in den Vordergrund stellen.

Auch bestimmte Zertifizierungen sind vermutlich dem Greenwashing zuzuordnen. So wird von einem Heimtextilien-Händler geworben, dass die Polster, Decken und Vorhänge nach dem Gebrauch zurückgenommen und „zu Nährstoff und Biogas kompostiert werden". Dies wird mit den Zertifikaten "Ocean Safe ${ }^{T M "}$ und "Cradle to Cradle Certified $^{T M " ~ a u s g e z e i c h n e t . ~ " D i e ~ a n-~}$ fallenden Nährstoffe sind die Basis für neue Rohstoffe zukünftiger Produkte". Wer glaubt wirklich, dass ein alter Polster nach Jahren des Gebrauchs genau diesem Händler zur „Kompostierung“ übergeben wird? Die Kompostierung oder die Verarbeitung von großen Mengen von Pölstern und Decken in Biogasanlagen ist aus abfalltechnischer Sicht ziemlich abstrus. Ein klassischer Fall für „Greenwashing"? (Kronen Zeitung 2020).
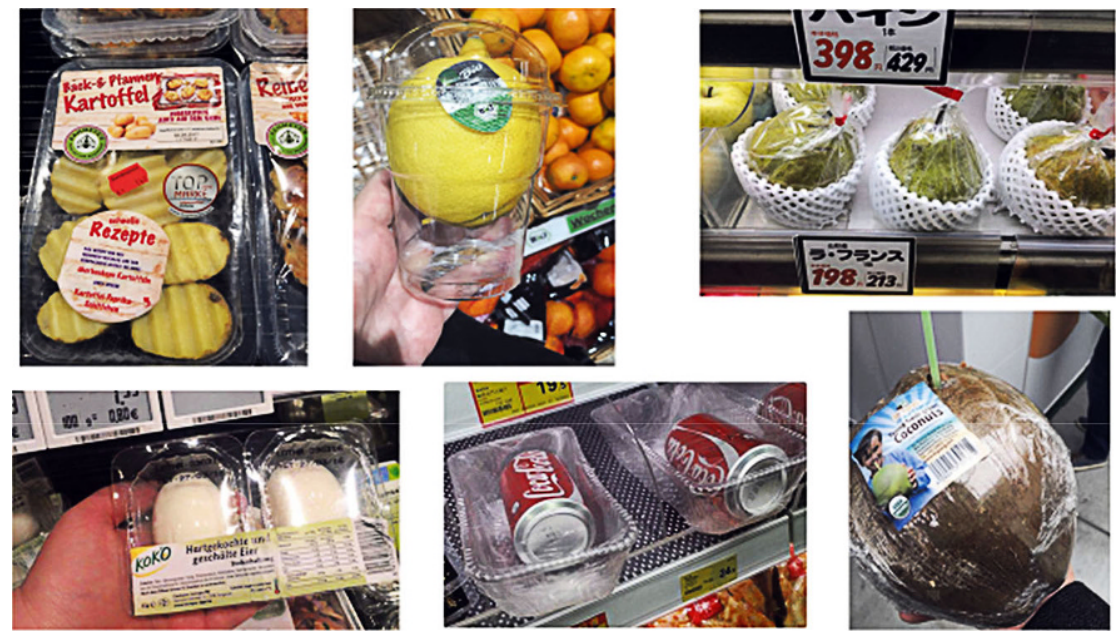

Abb. 2 Beispiele für „Dumme Produkte“(Pomberger 2019)

\subsection{Bio-Plastik}

Biobasierte Kunststoffe vermitteln dem Konsumenten den Eindruck, dass sie besonders umweltfreundlich seien und die Umweltprobleme der klassischen Kunststoffprodukte vermeiden. Vielfach wird mit der Auszeichnung ,kompostierfähig" geworben. Entscheidende Frage ist, ob unter realen Kompostierbedingungen tatsächlich ein vollständiger Abbau dieser Kunststoffe erfolgt. Die Kennzeichnung und Bewerbung von Einwegprodukten als biologisch abbaubar kann zu verstärktem Littering und zur Entsorgung über die Biotonne verleiten. Nur bestimmte Knotenbeutel im Lebensmitteleinzelhandel erfüllen die Voraussetzungen, um in Kompostieranlagen nicht als Störstoff zu gelten. Besonders problematisch sind Produkte aus oxo-abbaubaren Kunststoffen, die nicht kompostierbar sind und $\mathrm{zu}$ Mikroplastik zerfallen.

Für alle Abfälle aus biobasierten Kunststoffen besteht derzeit keine reale Recyclingfähigkeit im Sinne einer werkstofflichen Verwertung. Aufgrund der Vielfalt der eingesetzten Kunststoffarten und der sehr geringen Massenanteile in der Verpackung ist es in Sortieranlagen derzeit weder technisch noch wirtschaftlich möglich, daraus marktfähige Kunststoffkonzentrate zu erzeugen. Der abfallwirtschaftliche Nutzen der biobasierten Kunststoffe reduziert sich derzeit weitgehend auf ihre Treibhausgas-Neutralität in den Verbrennungsprozessen. 


\section{4 „Dumme Produkte“}

Die folgende Abbildung zeigt Beispiele für sogenannte "dumme Produkte“. Es sind dies Produkte bzw. ihre Verpackung, die so gestaltet sind, dass eine Verwertung keinesfalls möglich ist und auch ihr Nutzen einem umweltbewussten Bürger nicht zugänglich ist. Ausgewählte Beispiele zeigt Abb. 2.

\section{Was ist Recyclingfähigkeit?}

Die Recyclingfähigkeit lässt sich grundsätzlich für alle physischen Produkte feststellen. Sie ist ein individuelles Attribut und als graduelle Kennzahl Ausdruck und Instrument von Produktverantwortung. Die Bewertung der Recyclingfähigkeit wird im Wesentlichen von zwei Parametern bestimmt: der Beschaffenheit der Erzeugnisse und den realen Verwertungswegen nach Gebrauch.

Recyclingfähigkeit ist keine theoretische Eigenschaft. Richtig verstanden wird hiermit die stoffliche Eignung eines Erzeugnisses abgebildet, die im Rahmen etablierter Erfassungs- und Verwertungsstrukturen zur Schließung von Stoffkreisläufen beiträgt. Gerade für Verpackungen bestehen verschiedene Definitionen, welche sich in ihren Grundaussagen weitgehend decken:

Um als recyclingfähig zu gelten, müssen Produkte folgenden Kriterien entsprechen: Das eingesetzte Material wird durch länderspezifische sowie regionalspezifische Sammelsysteme erfasst und kann nach Stand der Technik sortiert werden. Weiters wird es in einem Recyclingprozess nach Stand der Technik verwertet. Die daraus gewonnenen Sekundärrohstoffe haben ein Marktpotenzial, um als Ersatz materialidenter Neuware verwertet zu werden. (ECR Austria 2020)

Recyclingfähigkeit in diesem Dokument bezieht sich im Unterschied zum Recyclingbegriff des KrWG immer auf ein hochwertiges und werkstoffliches Recycling. Diese Recyclingfähigkeit ist die grundsätzliche und graduelle Eignung einer Verpackung, nach Durchlaufen industriell verfügbarer Rückgewinnungsprozesse Neuware in werkstofftypischen Anwendungen zu substituieren. (Stiftung Zentrale Stelle Verpackungsregister 2019)

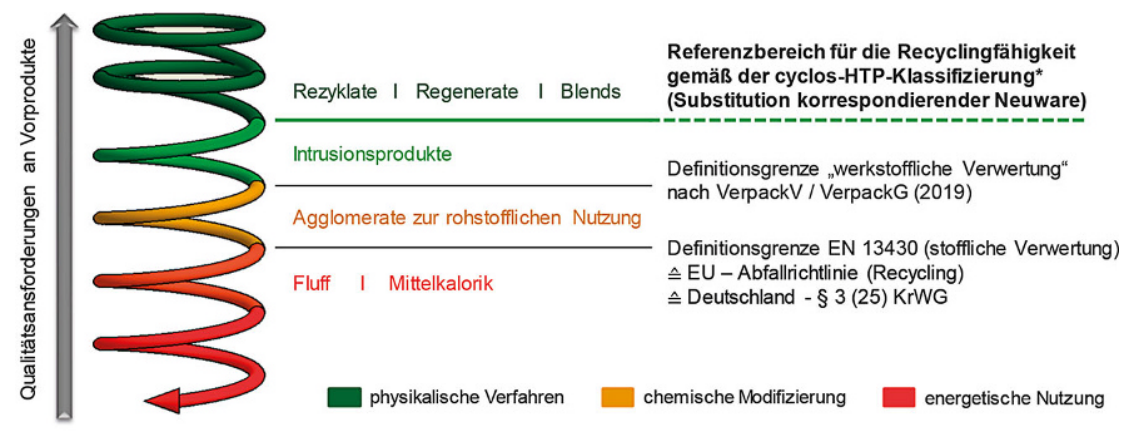

$\mathrm{a} \hat{=}$ (implizit) auch der D4R-Richtlinien

Abb. 3 Definition und Abgrenzung des Begriffes „Recycling“nach cyclos-HTP (2019). $\mathrm{a}_{\hat{=}}$ (implizit) auch der D4R-Richtlinien

„Recyclingfähigkeit ist die individuelle graduelle Eignung einer Verpackung oder eines Erzeugnisses, in der Nachgebrauchsphase tatsächlich materialidentische Neuware zu substituieren“; "tatsächlich" meint hierbei, dass Erfassungs- und Verwertungsstrukturen im industriellen Maßstab die Voraussetzung bilden. (cyclos-HTP 2019)

Kernpunkte bei diesen Definitionen sind einerseits die "werkstofftypische bzw. materialidente Substitution von Neuware“ und andererseits das „tatsächliche Durchlaufen von Recyclingprozessen". Das Material muss also "wirklich" gesammelt, sortiert und werkstofflich verwertet werden.

Die Anforderung der „materialidenten Substitution von Neuware" ist für Verpackungen durchaus nachvollziehbar und stellt hier ein anstrebenswertes Ziel dar. Es ist allerdings zu hinterfragen ob diese Anforderung grundsätzlich auch beim Recycling anderer Produkte aufrecht $\mathrm{zu}$ erhalten ist. Außerhalb des Verpackungsbereiches besteht eine viel größere Materialvielfalt, sodass die stoffliche Verwertung zu Rezyklaten, Regeneraten, Blends oder Legierungen, die in Standardanwendungen jeweils korrespondierendes Neumaterial ersetzen können, vielfach besonders schwierig ist und daher andere Anwendungen oder auch rohstoffliches Recycling erforderlich machen könnten.

Das herkömmliche Verständnis eines Closed-loop-Recyclings, bei dem rezyklierte Werkstoffe nicht nur korrespondierendes Neumaterial ersetzen, sondern darüber hinaus erneut in der identischen primären Anwendung zum Einsatz kommen können, wird in Abb. 3 durch eine zweite Ebene ergänzt, innerhalb der ggf. wiederum geschlossene Recyclingkreisläufe auf einem qualitativ niedrigeren Niveau realisiert werden können. Für Verpackungen werden Sekundärrohstoffe, die in neue Erzeugnisse eingebunden werden, die aber nicht das in der entsprechenden Anwendung typische Neumaterial ersetzen, sowie Verwertungsverfahren, bei denen Stoffe unmittelbar oder mittelbar energetisch genutzt werden, nicht als recyclingfähig klassifiziert.

\section{Recyclingfähigkeit aus Sicht der Abfallwirtschaft}

Der Begriff „Recyclingfähigkeit“ kann aus Sicht der Abfallwirtschaft definiert werden als Fähigkeit eines Produkts, nach einer getrennten Sammlung und/ oder abfalltechnischen Aufbereitung einer stofflichen Wiederverwertung zugeführt zu werden.

Am Ende des Lebenszyklus eines Produktes wird dieses zu Abfall und i.d.R. zu einem Teil eines Abfallgemisches. Für bestimmte Abfälle wurden getrennte Sammelsysteme eingeführt, für den Rest stehen gemischte Abfallarten wie z.B. gemischter Siedlungsabfall (Restmüll), Sperrmüll oder Gewerbeabfall zur Verfügung. Dabei zeigt sich, dass derzeit fast ausschließlich getrennt erfasste Altstoffe einem Recycling zugeführt werden. Für verwertbare Fraktionen in den gemischten Siedlungsabfällen gibt es derzeit keine verfügbare Rückgewinnung mit Ausnahme von Metallfraktionen. Umfangreiche Forschungsarbeit (u.a. an der Montanuniversität) mit gemischten Abfällen zeigt, dass die Sortierung von gemischtem Siedlungsabfall hohe Investitionen erfordert und leider nur geringes Aus- 


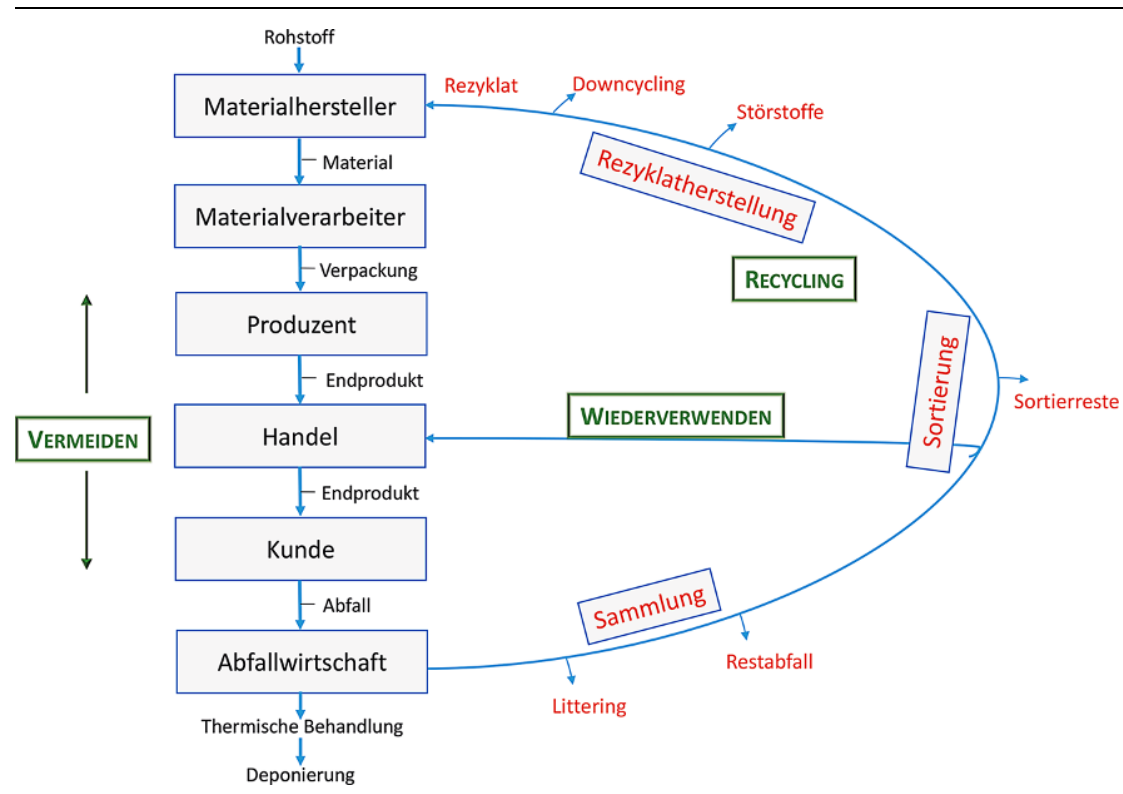

Abb. 4 Wertschöpfungskette von Kunststoffen durch Vermeiden, Vorbereitung zur Wiederverwendung und Recycling unter Berücksichtigung der kreislaufwirtschaftlichen Rückführung ins selbe Produkt (Wellacher et al. 2020)

bringen sowie Fraktionen mit hoher Verschmutzung generiert. Der Aufwand wird derzeit durch die Wertstofferlöse nicht gerechtfertigt.

Für die getrennt erfassten Altstoffe führt die Abfallwirtschaft die Prozessschritte Sammlung, Sortierung und Aufbereitung durch und erzeugt i.d.R. Wertstoffkonzentrate. Diese werden werkstoffspezifisch in Recyclinganlagen zu Rezyklaten, Regeneraten, Blends oder Legierungen verarbeitet und als Sekundärprodukte vermarktet. Abb. 4 zeigt, dass die Abfallwirtschaft nur einen Teilbereich der Wertschöpfungskette eines Produktes abdeckt. Das eigentliche Recycling stellt einen eigenen industriellen Prozess dar, der sich meist außerhalb der klassischen Abfallwirtschaft befindet. Die Mengenströme und Qualitäten, die am Ende im Recycling landen, werden aber weitgehend durch die Aktivitäten der klassischen Abfallsammler und Behandler bereitgestellt.

Ein maßgeblicher Einflussfaktor auf die Recyclingfähigkeit eines Produkts definiert der reale abfallwirtschaftliche Prozess, dem es zugeführt wird. Heutige Aufbereitungs- und Sortieranlagen stellen komplexe Prozessketten dar, in denen neben einer (selektiven) Vorzerkleinerung, Klassierung, Windsichtung sowie Magnet- und Wirbelstromscheidung, meist sensorgestützte Sortieraggregate eingesetzt werden. Aufbau und Zusammensetzung solcher
Prozessketten sind entscheidende Kriterien für den Verbleib des Abfalls bzw. ehemaliger Produkte, in den jeweils entstehenden Outputfraktionen (z.B. Restfraktion, Fraktion zur thermischen cling). Ein recyclinggerechtes Produktdesign kann also nur vorliegen, wenn nicht nur die prinzipielle Recyclingfähigkeit der einzelnen Bestandteile eines Produkts gegeben ist, sondern auch die Rückgewinnung aus dem jeweiligen abfallwirtschaftlichen System, inklusive Sammlung, Aufbereitung, Vorsortierung und dem eigentlichen Recycling, erfolgen kann. Insbesondere der Aspekt der Vorbehandlung (Aufbereitung und Vorsortierung von Abfallströmen) wird derzeit aber nicht ausreichend bei der Feststellung eines recyclinggerechten Produktdesigns berücksichtigt.

Darüber hinaus stehen oftmals die Anforderungen von Kunststoffrecyclern, der Aufbau der abfallwirtschaftlichen Systeme und die technischen Möglichkeiten in Aufbereitungs- und Sortieranlagen in einem Spannungsverhältnis, wodurch die tatsächliche Rezyklierbarkeit eines Produkts weiter beeinflusst wird. Auch diese Zusammenhänge sind also für Bewertung eines recyclinggerechten Produktdesigns wichtig.

Die Recyclingfähigkeit von Produkten (außerhalb der Verpackungen) wird derzeit nicht standardisiert untersucht. D.h. es gibt keine Testverfahren, die Verwertung, Vorkonzentrate fürs Recy- überprüfen, ob ein Produkt (als Abfall) in einer technischen Sortieranlage nach Stand der Technik erkannt werden kann, durch Sortiermaschinen ausgeschleust und anschließend rezykliert werden kann.

Die Beurteilung von Produkten hat bisher lediglich im Verpackungsbereich zu "labels" geführt, die aber derzeit eher auf Basis von Checklisten und einfachen Entscheidungsheuristiken die Recyclingfähigkeit beurteilen. In der Regel basieren diese Entscheidungen auf Geometrie und Werkstoff. Die realen technischen Möglichkeiten der Sortiertechnologien der Abfallwirtschaft werden dabei bisher, auch international, noch nicht mit Testverfahren untermauert. Dies stellt einen grundsätzlichen methodischen Mangel dar. Es fehlt international und damit natürlich auch in Österreich ein Verfahren, welches Produkte auf ihre Detektierbarkeit, Sortierbarkeit und Ausschleusbarkeit und damit auf ihre reale Recyclingfähigkeit in der Abfallwirtschaft untersucht und testet.

Um die bisherigen Recyclingquoten zu erhöhen, muss neben der Verbesserung von Aufbereitungstechnologien vorrangig die Recyclingfähigkeit von Produkten gesteigert werden. Derzeit ist die Bewertung der Recyclingfähigkeit von Produkten jedoch nicht eindeutig geklärt. Die Recherche der aktuell am Markt existierenden Label/Prüfzeichen zeigt sehr deutlich, dass es derzeit kein für den Massenmarkt einsetzbares und den EU-Vorgaben aus dem Circular Economy Package vollständig entsprechendes Label/Prüfzeichen für Recyclingfähigkeit gibt. Die Anzahl der Label/Prüfzeichen ist beschränkt und jene, die inhaltlich den zukünftigen EUVorgaben am ehesten entsprechen, sind wissenschaftlich bzw. anwendungsseitig sehr komplex aufgebaut.

Inhaltlich decken sie, wenn überhaupt, meist nur den "Recycling-Loop“ aus dem „MacArthur Circular Econo$m y^{\prime \prime}$-Diagramm ab. Somit wird zwar die prinzipielle Recyclingfähigkeit eines Materials bewertet, jedoch nicht der Einfluss, den die realen abfallwirtschaftlichen Systeme auf die Recyclingquote von Wertstoffen haben. Es gibt also derzeit keine einheitlichen, groß angelegten Labels/Prüfzeichen zur Bestimmung der Recyclingfähigkeit von Produkten, obwohl diese von den Stakeholdern entlang der Kunststoffwertschöpfungskette verlangt werden. 
Viele Produkte sind somit gar nicht oder nur teilweise recyclingfähig.

Zudem bestehen in vielen Bereichen noch keine (abfallwirtschaftlichen) Vorgaben, um die Recyclingfähigkeit von Produkten zu definieren. Die rechtlichen Rahmenbedingungen der EU fordern jedoch auch die intensive Einbindung der Abfallwirtschaftsund Recyclingbranche neben der produzierenden Industrie, da diese auf der einen Seite Sekundärrohstoffe und Rezyklate herstellen und auf der anderen Seite Qualitätsstandards für den Einsatz dieser in der Produktion erfüllen müssen. Somit ist es essenziell, die Recyclingfähigkeit von Produkten mit Produzenten und abfallwirtschaftlichen Stakeholdern gemeinsam zu bewerten, um eine tatsächliche Verbesserung des Ist-Stands zu ermöglichen. Konkret bedeutet dies die Verbindung des Knowhows beider Akteursgruppen, welche derzeit - jeder für sich - in seinem Marktumfeld mit den dort geltenden Voraussetzungen mehr oder weniger autonom arbeiten. Die Entwicklung solcher gemeinsamen Standards und der gleichzeitige Aufbau eines gemeinsamen Verständnisses zwischen Verund Entsorgungsseite zur Erfüllung der neuen EU-Vorgaben wäre anzustreben.

\section{Zusammenhang von Recyclingquote und recyclingfähigen Produkten}

Um die Recyclingquoten zu erhöhen, stehen grundsätzlich mehrere Strategien zur Verfügung:

1. Mehr sammeln: dadurch werden auch mehr verwertbare Abfälle gesammelt.

2. Genauer sortieren: durch höhere Sortiertiefe steigert sich das "Inhaltsausbringen“, d.h. ein höherer Anteil des verwertbaren wird tatsächlich durch Sortierung erfasst und als Konzentrat ausgeschleust.

3. Anteil des Verwertbaren steigern: wenn der Anteil der recyclingfähigen Partikel im Sortiergut (Wertstoffpotenzial) steigt, so kann durch bestehende Sortiertiefe ein höheres Masseausbringen erreicht werden.

Ganz vereinfacht lässt sich der Erfolg der Sortiertechnik auf folgende Grundsätze reduzieren:

„Was nicht gesammelt wurde, kann ich nicht sortieren."

„Was nicht drin ist, kann ich nicht rausholen.“
„Was nicht erkennbar ist, kann ich auch nicht rausholen."

Für den Bereich der Kunststoffverpackungsabfälle wurde in den letzten Jahren die Sortiertiefe (entspricht weitgehend dem technischen Begriff des Inhaltsausbringens) in den Sortieranlagen deutlich gesteigert (von ca. 35\% auf ca. $50 \%$ ). Eine weitere Steigerung scheint nur machbar, wenn das Wertstoffpotenzial (d.h. der Anteil an recyclingfähigen Teilen) gesteigert wird. Dies kann von der Abfallwirtschaft nicht beeinflusst werden, es ist die Aufgabe der Hersteller und des Handels, den Einsatz recyclingfähiger Verpackungen $\mathrm{zu}$ steigern. Geeignete Lizensierungssysteme, die recyclingfähige Produkte bevorzugen, würden diese Entwicklung sicherlich sehr positiv unterstützen.

Für den Bereich stoffgleicher Nichtverpackungen, also grundsätzlich recyclingfähiger Verpackungen, die allerdings aus wirtschaftlichen Gründen nicht sortiert und verwertet werden, sollten Rahmenbedingungen geschaffen werden, die diese bereits gesammelten und verwertbaren Abfälle im Kreislauf halten.

Für die Vielzahl an Produkten außerhalb der Verpackungen bestehen derzeit mit einigen Ausnahmen (Elektroaltgeräte, Altfahrzeuge, Batterien) keine Sammel- und Verwertungsquoten. Natürlich ist eine Quote ein starkes gesetzliches Werkzeug, um Herstellerverantwortung verpflichtend $\mathrm{zu}$ machen. Wenn in der Aufbereitung keine Stoffströme mit monetärem Wert entstehen, wird auch kein Recycling betrieben, da es lediglich einen wirtschaftlichen Nachteil mit sich bringt. Solange keine verpflichtenden Quoten bestehen, können Hersteller und Handel das Qualitätsmerkmal „recyclingfähig" völlig frei verwenden. Dass damit bereits heute Täuschung und Missbrauch erfolgen, ist bei der Sichtung von "recyclingfähigen" Produkten offensichtlich. Wirklich recyclingfähige Produkte haben zumindest das Potenzial, getrennt gesammelt und verwertet zu werden. Neue Geschäftsmodelle in Kooperation zwischen Herstellern/ Handel und Abfallwirtschaftsunternehmen könnten dadurch entstehen. Die Recyclingfähigkeit der Produkte ist dafür die Grundvoraussetzung.

\section{Stufenmodell der \\ Recyclingfähigkeit}

Die theoretische Recyclingfähigkeit prüft die Produkte im Originalzustand und in der Regel auf Basis des Materials. Eine Einstufung als recyclingfähig bedeutet dann auch nicht, dass dieses Produkt als Abfall tatsächlich recyclingfähig ist. Dazu gehört noch die Prüfung der technischen Erkennbarkeit durch geeignete Sensoren und die Ausschleusbarkeit in realen Maschinen und Anlagen. Wird dies berücksichtigt, so können wir eine technische Recyclingfähigkeit feststellen. Recycling wäre dann technisch machbar. In einem regionalen Kontext kann dann die Frage beantwortet werden, ob das Produkt tatsächlich gesammelt wird, ob die regionale Sortieranlage dieses Produkt als Wertstoff tatsächlich ausschleust und ob für diese Fraktion ein realer Markt besteht. Des Weiteren ist zu klären, ob auch das nachfolgende Recyclingverfahren diesen Wertstoff weitgehend in sein Sekundärprodukt überführt. Sind diese Voraussetzungen gegeben so besteht reale Recyclingfähigkeit (siehe Abb. 5).

Im Extremfall kann die theoretische Recyclingfähigkeit hoch, aber die technische und damit auch die reale Recyclingfähigkeit Null sein. Auch der Fall, dass die theoretische und die technische Recyclingfähigkeit hoch sind, aber die reale Recyclingfähigkeit Null ist, ist durchaus möglich. Dies wäre der Fall, wenn ein recyclingfähig designtes Produkt, das sortier- und recyclingfähig ist, in einer Region einfach nicht gesammelt wird.

\section{Recyclingfähigkeit messbar machen}

\subsection{Bestehende Methoden und Labels}

Bislang existieren zwar verschiedene Standards für die Bewertung der Recyclingfähigkeit von Verpackungen:

- DSD - RecyclingCOMPASS Design4Recycling Richtlinien;

- bifa - Recyclingfähigkeit von Verpackungen - Bewertungskatalog;

- Mindeststandard für die Bemessung der Recyclingfähigkeit von systembeteiligungspflichtigen Verpackungen gemäß $§ 21$ Abs. 3 VerpackG (Zentrale Stelle Verpackungsregister 2019);

- cyclos-HTP - Anforderungs- und Bewertungskatalog zur EU-weiten Zertifizierung (Cyclos-HTP 2019); 


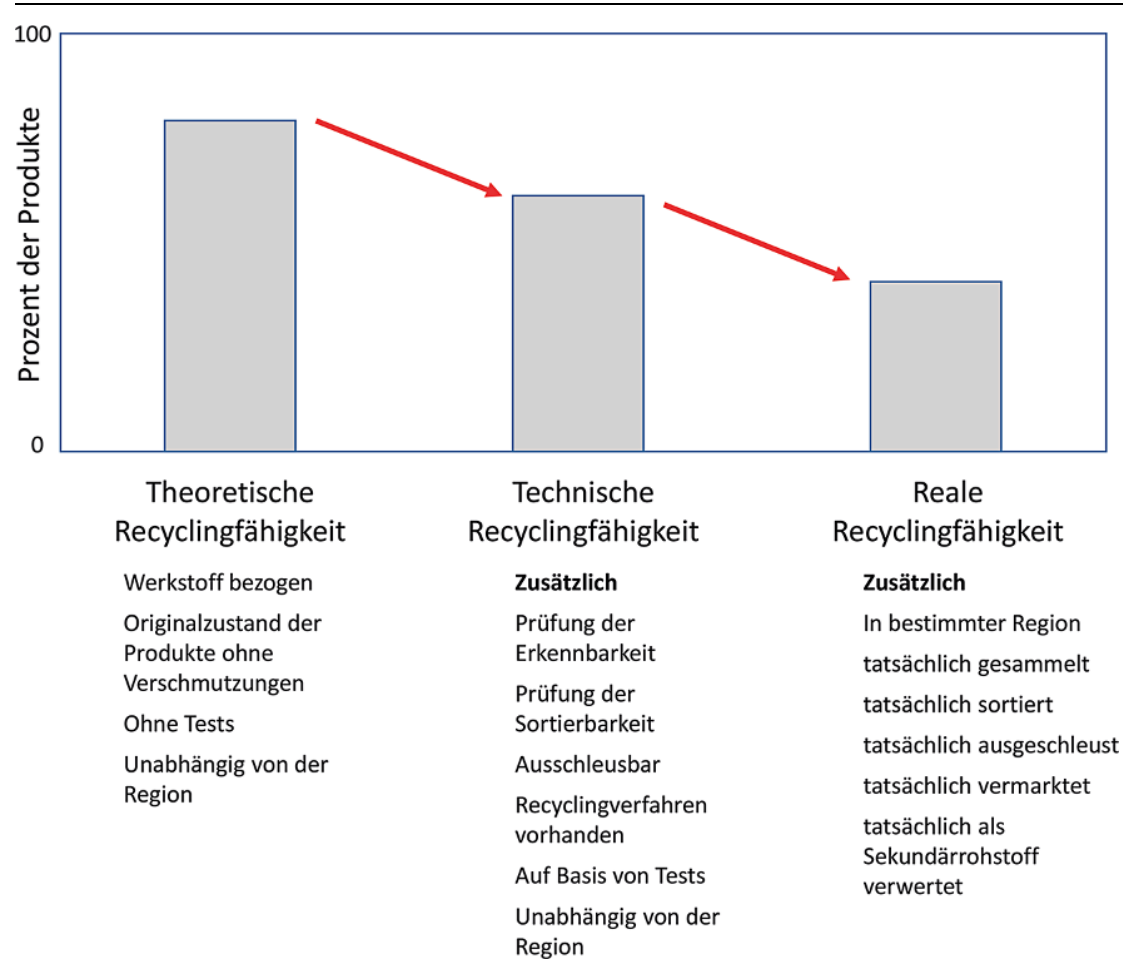

Abb. 5 Zusammenhang zwischen theoretischer, technischer und realer Recyclingfähigkeit

- FH Campus Wien - Circular Packaging Design Guideline (Tacker und Krottendorfer 2020);

- RecyClass recyclability evaluation protocol for HDPE containers;
In vielen Verfahren fehlen derzeit der praktische Test der realen Sortierbarkeit und die Beurteilung der realen Recyclingfähigkeit. Als positives Beispiel wird die Vorgangsweise nach cyclosHTP dargestellt. Aus dem Ablaufschema ist erkennbar, dass wesentliche Anforderungen an die Recyclingfähigkeit abgedeckt werden und insbesondere das Vorhandensein von Erfassungsund Verwertungsstrukturen bewertet wird (siehe Abb. 6).

Bislang bestehen keine Methoden oder standardisierte Verfahren, die es erlauben, eine Bewertung der realen Recyclingfähigkeit spezifischer Produkte (außerhalb des Verpackungssektors) vorzunehmen. Dazu ist es erforderlich, State-of-the-Art-Sensortechnologien zu verwenden, um in praktischen Tests die Klassifizierung mittels Algorithmen, die für die Sortierung geeignet sind, die Differenzierbarkeit von Produkt und restlichem Abfallstrom sicherzustellen. Bislang wird dies nicht transparent und nachvollziehbar umgesetzt, wodurch auch die Übertragbarkeit auf heutige Sortieranlagen nicht gegeben ist. Nur durch die Entwicklung von standardisierten Routinen kann die praktische Prüfmöglichkeit entwickelt und so eine für die Wirtschaft ausreichende Basis geschaffen werden. Darüber hinaus müssen solche Methoden auch für Produkte außerhalb des Verpackungssek-

\section{Recyclingfähigkeit nach Institut cyclos-HTP (CHI)}

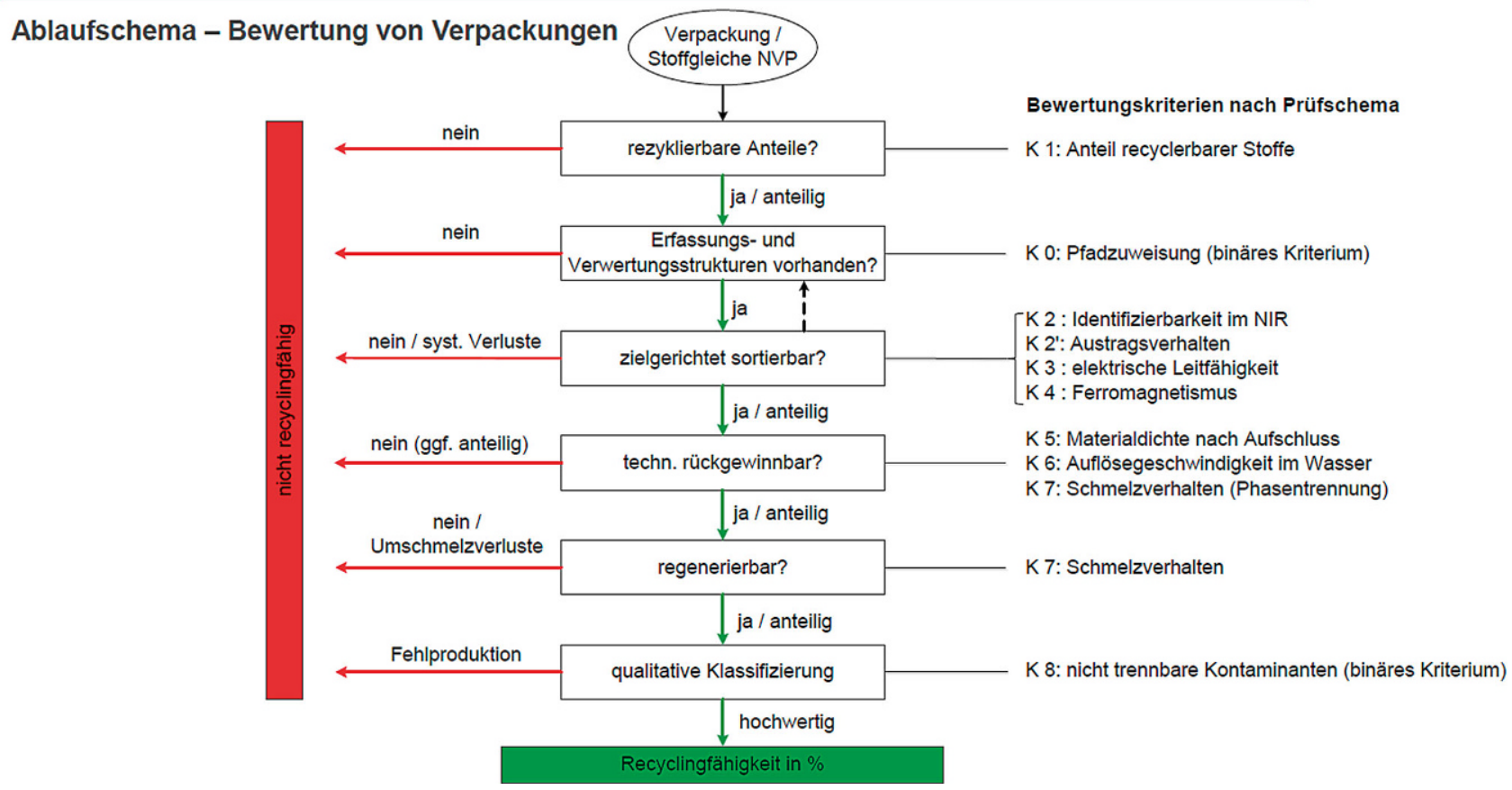

Abb. 6 Ablaufschema der Bewertung der Recyclingfähigkeit nach cyclos-HTP (2019) für Verpackungen 


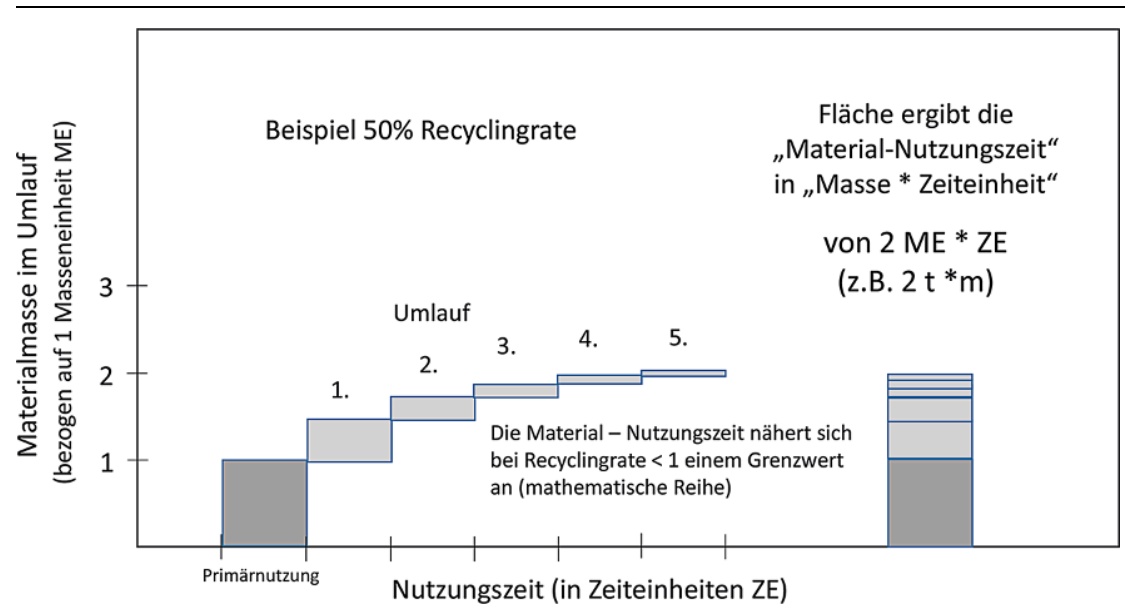

Abb. 7 Prinzipielle Darstellung der „Material-Nutzungszeit“ am Beispiel eines Produktes, das mit einer $50 \%$-Recyclingrate verwertet wird

tors anwendbar sein. Um die damit einhergehenden Anforderungen zu erfüllen, ist es notwendig neue Ansätze zu finden und auszuarbeiten.

\subsection{Methode der End-of life-Material- Nutzungszeit}

In Analogie zur Bewertung des Transportaufwandes in der Logistik - hier wird der Transportaufwand in „Tonnen * Kilometern" verglichen - könnte in der Abfallwirtschaft ein ähnlicher Vergleichswert verwendet werden. Die zwei Dimensionen „Materialumlauf" und „Nutzungszeit" könnten zu einer Kenngröße „Material-Nutzungszeit" verbunden werden. Abb. 7 zeigt das Prinzip dieser Bewertung.

Dieses Konzept unterstellt, dass Material möglichst lange genutzt werden soll. Dies kann durch hohe Recyclingrate, durch möglichst viele Kreisläufe, aber auch durch lange Nutzungsdauer erreicht werden. Wenn die Recyclingrate gering ist, so wird nur wenig des Ursprungsmaterials in den nächsten Kreislauf übergeführt. Wenn die Nutzungsdauer des Produktes kurz ist, so wird trotz mehrerer Kreisläufe das Material nur geringe Zeit verwendet. Viele Kreisläufe mit geringer Nutzungszeit können durchaus eine geringere „Material-Nutzungszeit" ausweisen als ein Produkt, das zwar nur einmal verwendet wird, aber eine sehr lange Nutzungsphase aufweist. Die zentralen Parameter, die die „Material-Nutzungszeit" beeinflussen sind:

- die Recyclingrate,

- die Nutzungszeit in jedem Kreislauf und

- die Anzahl der Umläufe.
Es könnte damit die Dauer bewertet werden, die ein Sekundärrohstoff tatsächlich im Umlauf ist, und damit Nutzen generiert. Dazu folgende Beispiele (vgl. Abb. 8):

- Ein Abfall wird nicht recycelt. Das Material wird also nur einmal verwendet. Die Material-Nutzungszeit ist von der Nutzungszeit dieses Pro-

- Ein Abfall wird recycelt, aber nur einmal in einem geringwertigen Einmalprodukt eingesetzt. Ein gewisser Prozentsatz des ursprünglichen Materials geht in das neue Einmalprodukt. Dieses wird eine bestimmte Zeit verwendet und dann $\mathrm{z}$. B. thermisch verwertet. Die Material-Nutzungszeit verlängert sich um das Produkt aus Recyclingrate mal neuer Nutzungszeit.

- Ein Abfall wird recycelt, aber mehrfach in einem vergleichbaren Produkt eingesetzt. Die Material-Nutzungszeit ist nun die Summe aller Umläufe des Produktes mal neuer Nutzungszeit.

Diese Methode könnte sich eignen, verschiedene Produkte mit und ohne Recycling in Bezug auf ihren Materialumlauf $\mathrm{zu}$ vergleichen. In diesem Zusammenhang wird darauf verwiesen, dass das Konzept des „Materialkreislaufs" bereits verwendet wird, allerdings wird hier nur betrachtet, wieviel des Materials im Kreislauf geführt wird. Die zweite Dimension, nämlich die Dauer der Nutzung, wird dabei noch nicht berücksichtigt. Abb. 9 zeigt die Materialkreisläufe in Abhängigkeit von der Kreislaufrate. Diese Kurve zeigt sehr deutlich, dass in Bezug auf die duktes bestimmt.
Materialeffizienz nur eine sehr hohe Recyclingrate mit der Mehrwegnutzung mithalten kann.

\subsection{DIGITAL Waste Characterisation $\mathrm{LAB}$}

Am Lehrstuhl für Abfallverwertungstechnik und Abfallwirtschaft der Montanuniversität Leoben wurde 2017 in einen Versuchsstand für sensorgestützte Erkennung und Sortierung (NIR/VIS/EM Rutschensortierer der Binder AG) (Abb. 10) investiert. Mit dieser Forschungsinfrastruktur wurden insbesondere Oberflächeneffekte (z.B. Verschmutzungen) und ihre Auswirkung auf die Erkennbarkeit sowie grundlegende Untersuchungen zur Sortierfähigkeit verschiedener Abfallarten untersucht.

Derzeit laufen die Planungen zum Aufbau eines „DIGITAL Waste Characterisation LABs“ (Inbetriebnahme 1/2021) (Abb. 11), mit dem die technische Infrastruktur zur Entwicklung von Testmethoden zur Beurteilung der technischen Sortierbarkeit und Recyclingfähigkeit aufgebaut werden soll. Gemeinsam mit den Möglichkeiten des neuen Technikums sollen reale Sortier- und Aufbereitungsprozesse beurteilt werden können. Ziel ist es, neue Testmethoden für die Prüfung von Produkten in Bezug auf Recyclingfähigkeit zu entwickeln und an ausgewählten Produkten zu testen.

In einem neuen Forschungsprojekt soll die Entwicklung einer standardisierten Methode zur Bewertung der Recyclingfähigkeit von Produkten unter Berücksichtigung der realen abfallwirtschaftlichen Systeme erfolgen. Der Fokus liegt hierbei auf Produkten, die vollständig oder zu großen Teilen aus Kunststoffen bestehen, da diese in vielen Abfallströmen verbreitet sind, einen signifikanten Anteil des heute anfallenden Abfalls ausmachen und aufgrund ihrer chemischen Beschaffenheit besonders hohe Anforderungen an die Reinheit von Vorkonzentraten für das Recycling stellen.

So soll es durch standardisierte Testverfahren auf Basis von Sensortechnologien möglich werden, quantifizierbare Aussagen zur Recyclingfähigkeit von Produkten zu treffen. Diese Methodenentwicklung zur Bewertung der Recyclingfähigkeit, unter Berücksichtigung des jeweiligen Recyclingprozesses ist essenziell, um die Forcierung von tatsächlich recyclingfähigen Produkten 

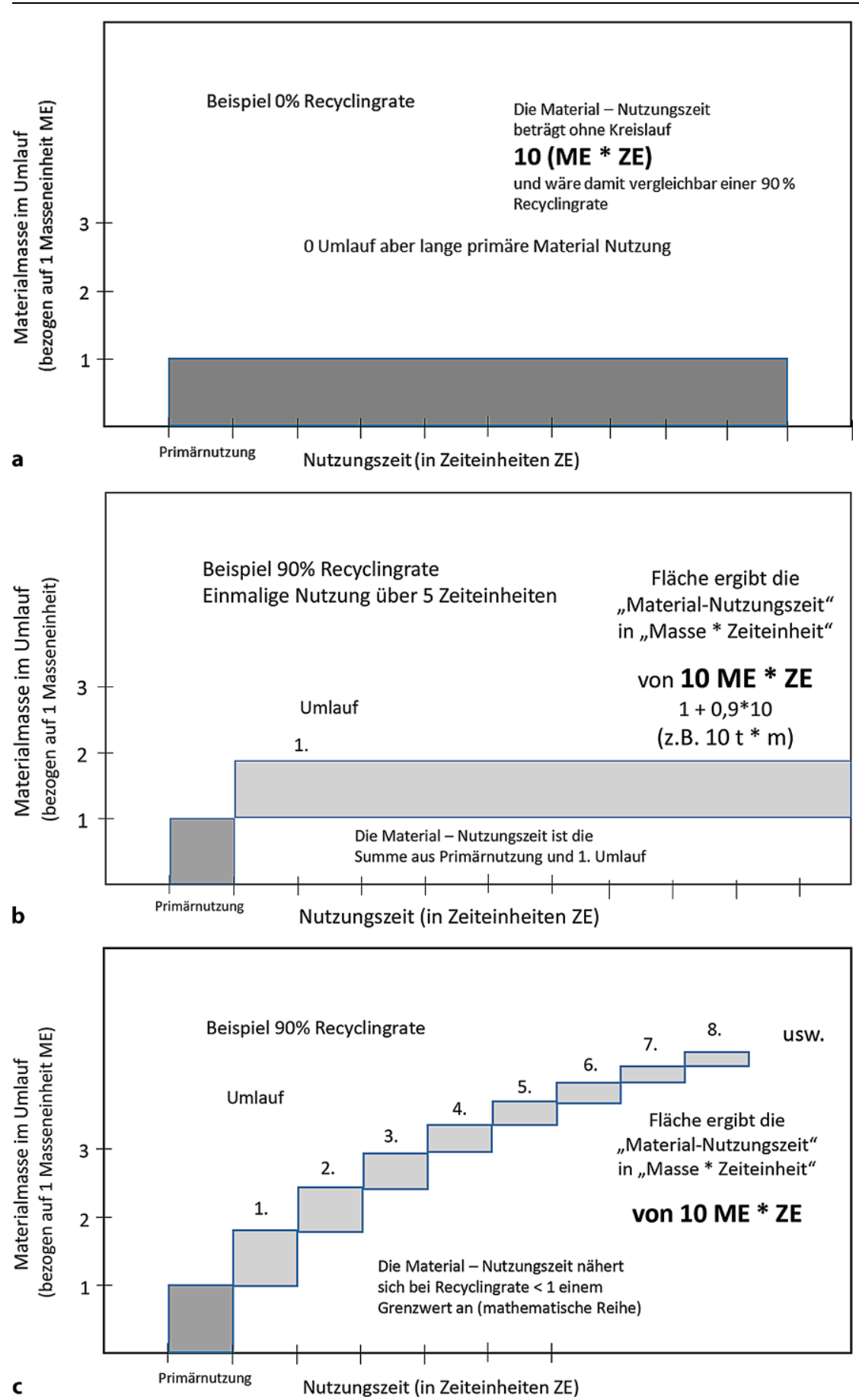

Abb. 8 Verschieden Beispiele für die Anwendung der „Material - Nutzungszeit“. a Material ohne Umlauf und langer Nutzungszeit. b Material mit einmaligem Umlauf und langer Nutzungszeit im 1. Umlauf. c Material mit hohem Umlauf und hoher Recyclingrate

und daraus resultierende Ressourcenund Treibhausgas-Einsparungen zu ermöglichen. Ziel ist die Implementierung der zu entwickelnden Methodik in bestehende Verfahren und Labels/ Prüfzeichen zur Bewertung der Recyclingfähigkeit von Produkten unter

Einbeziehung der Aufbereitung und Sortierung der relevanten Abfallströme.

\section{Lösungsansätze}

Folgende Lösungsansätze und Ideen sollten die verbesserte Recyclingfähigkeit von Produkten fördern. Nur diffe- renzierte Maßnahmen auf verschiedenen Ebenen und durch verschiedene Stakeholder werden $\mathrm{zu}$ einem Erfolg führen können. Entscheidender Bedeutung kommt dabei den Lenkungsmaßnahmen und den gesetzlichen Rahmenbedingungen $\mathrm{zu}$.

\subsection{Forderungen des ÖWAV}

Im ÖWAV-Positionspapier „Strategien der österreichischen Recycling- und Abfallwirtschaft" (ÖWAV 2018) wurden Handlungsaufforderungen definiert, um Ökodesign voranzutreiben. Diese Unterlage wurde in einem zweijährigen Prozess unter Einbeziehung von Experten und Stakeholdern entwickelt und soll insbesondere der Politik und Meinungsträgern als fachliche Grundlage dienen.

„Kunststoffhersteller und -verarbeiter: Primäres Ziel des Kunststoffrecyclings ist die Herstellung von Sekundärrohstoffen für hochwertige (gleichwertige) Produkte. Mithilfe erhöhter und einheitlicher Qualitätsstandards in der Herstellung von Kunststoffen werden die Erzeugung hochwertiger Rezyklate und ihr Einsatz als Sekundärrohstoff gefördert. Dazu ist es erforderlich, klare Vorgaben hinsichtlich der Inhaltsstoffe (Schadstoffreduktion) bzw. der Zusammensetzung von Kunststoffen und Kunststofferzeugnissen sowie deren Recyklatanteil festzulegen, die von der produzierenden Wirtschaft einzuhalten und auszuweisen sind. Die EUKunststoffstrategie fordert zudem die Recyclingfähigkeit aller Kunststoffverpackungen bis zum Jahr 2030.

\section{Bau- und Werkstoffindustrie, Planer:}

Der Trend $\mathrm{zu}$ nicht recyclingfähigen Materialverbunden konterkariert die Anstrengungen der österreichischen Recyclingwirtschaft, das Baustoffrecycling weiter $\mathrm{zu}$ etablieren. Recyclingfreundliche Bauprodukte, u.a. leicht trennbare Komponenten, sind in der Produktion, Planung und Ausführung bevorzugt einzusetzen. Entsprechende Förderinstrumente sind anzupassen und umzusetzen.

Fahrzeugindustrie: Die Verwertung von Verbundmaterialien und Spezialwerkstoffen, wie z.B. Carbonfasern, stellt die Recycling- und Abfallwirtschaft derzeit vor große Herausforderungen. Neue Werkstoffe und Verbunde müssen daher auch unter Berücksichtigung ihrer Recycling- und Demontagefähigkeit entwickelt werden (Produzentenverantwortung). 


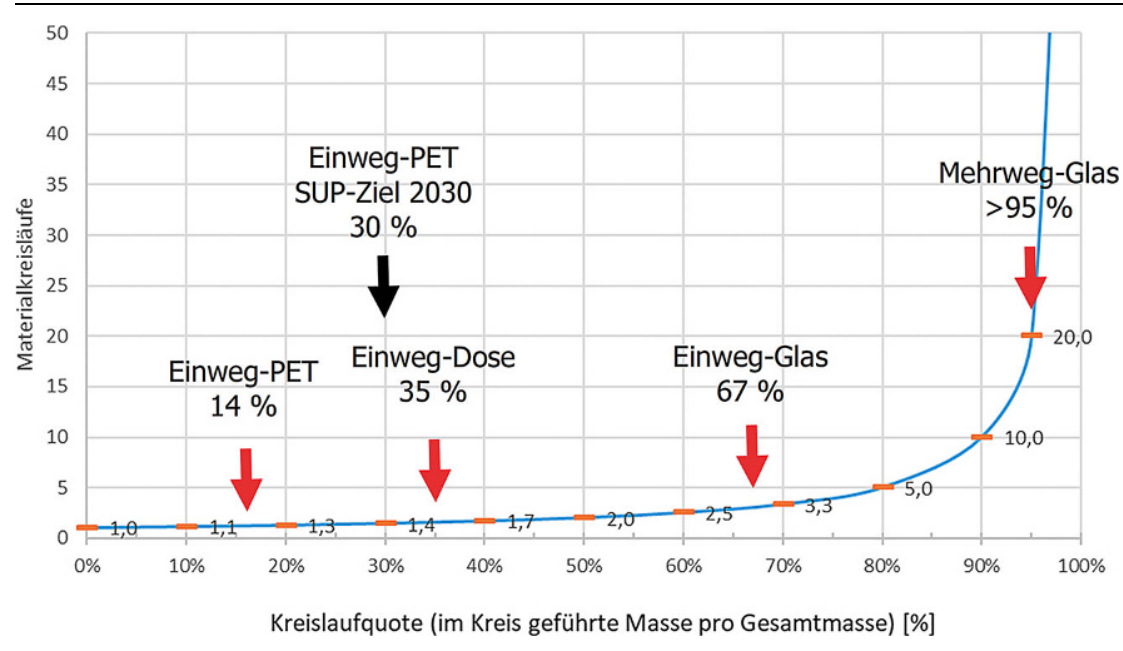

Abb. 9 Zusammenhang der Vermeidungs-Kennziffer „Materialkreisläufe“ mit der Recycling-Kennziffer „Kreislaufquote“ auf Basis des Materialgewichtes und die Kreislaufquoten für PET-Getränkeflaschen, Getränkedosen und Glasflaschen in Österreich (Wellacher et al. 2020)

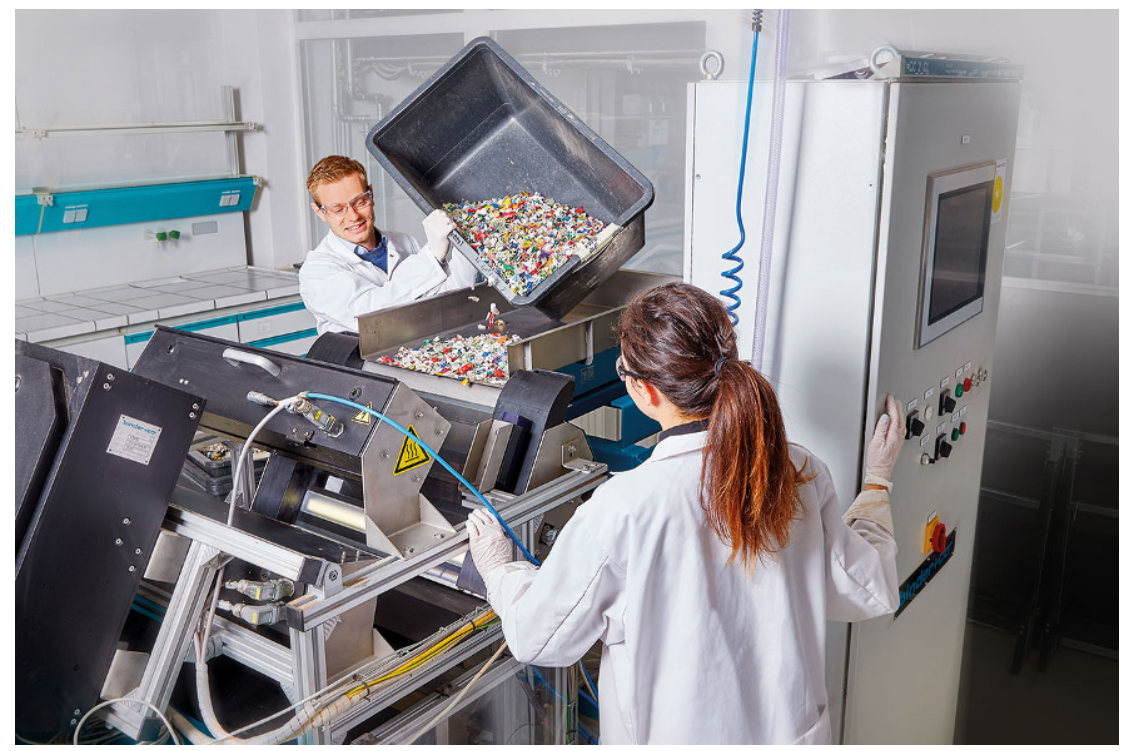

Abb. 10 Versuchstandfürsensorgestützte Erkennungund Sortierungam Lehrstuhl für Abfallverwertungstechnik und Abfall wirtschaft (AVAW) der Montanuniversität Leoben

Elektro- und Elektronikindustrie: Miniaturisierung und Technologiekonvergenz haben im Elektronikbereich zu hoher Komplexität und Werkstoffvielfalt geführt. Dennoch sollen die in Elektround Elektronikgeräten verbauten Stoffe für eine Verwertung verfügbar bleiben. Auch das Recycling von kritischen Rohstoffen (z.B. seltene Erden) trägt zur Ressourcenschonung bei. Inhaltsstoffe und Bauteile müssen bekannt, erkennbar und sortenrein erfassbar sein. Der mit der Energiewende verbundene Umstieg auf erneuerbare Energieträger (einschließlich E-Mobilität) ist wohl logisch, dass reale Recyclingfähigkeit (und nicht nur die theoretische) beim Lizenzentgelt bevorzugt werden muss. Differenzierte Lizenztarife schaffen einen Anreiz für die recyclinggerechte Produktgestaltung und hohen Einsatz von Recyclingrohstoffen. Nicht recyclingfähige Produkte sollten in eine schmerzhaft teurere Lizenzierungsklasse fallen. Damit kann bei den Produzenten und im Handel ein wirkungsvoller Lenkungseffekt erzielt werden. Recyclingfähigkeit bei Produkten privilegieren und nicht recyclingfähige Produkte teurer machen, das erscheint als wichtigste Lenkungsmaßnahme.

\subsection{Recyclingfähigkeits-Label}

Recycling Labels werden von verschiedenen Organisationen angeboten. Es wäre eine überlegenswerte Idee, dass die Abfallwirtschaft selbst z. B. durch ihre Interessensvertretungen ein eigenes Recycling-Label anbietet. Dieses könnte die tatsächliche "reale Recyclingfähigkeit" adressieren. Wer, wenn nicht die kommunalen und gewerblichen Abfallwirtschaftsunternehmen, weiß am besten, ob ein Produkt/Abfall tatsächlich gesammelt, sortiert und verwertet wird.

\subsection{Sensor-based Sorting-Technologie}

Die sensorgestützte Sortierung nimmt in State-of-the-Art-Aufbereitungsprozessen eine besondere Rolle ein: Vorgeschaltete Aggregate, wie z. B. Shredder, Siebe und Windsichter, reichern zwar Wertstoffe in bestimmten Materialströmen einer Aufbereitungs- oder Sortieranlage an, die eigentliche Erzeugung von Konzentraten für das Recycling erfolgt aber insbesondere für Kunststoffe durch sensorgestützte Sortieraggregate. Die mit den eingesetzten Technologien einhergehenden Limitierungen sind also für die realistische Bewertung eines recyclinggerechten Produktdesigns essenziell. Der verstärkte Einsatz dieser Technologien, insbesondere in neu $\mathrm{zu}$ errichtenden Sortieranlagen, wird die Möglichkeiten der Erkennung und Sortierung und damit die Sortiertiefe deutlich steigern.

\subsection{Ausbau der Erweiterten Herstellerverantwortung}

Eine wichtige Lenkungsmaßnahme ist die Bevorzugung von recyclingfähigen Produkten bei den Lizenzentgelten von Sammel- und Verwertungssystemen. Es
Erweiterte Produzentenverantwortung (,Extended Producer Responsibility“, EPR) dehnt die Verantwortung des Her- 


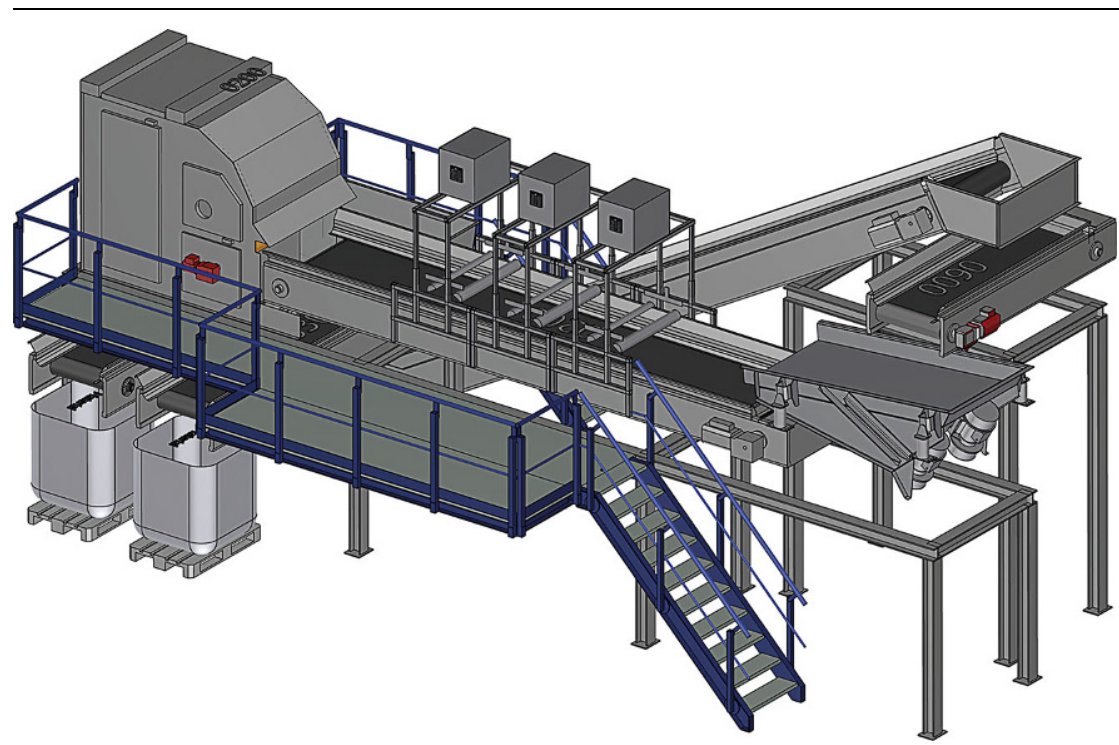

Abb. 11 Geplante Technikumsanlage im „DIGITAL Waste Characterisation LAB“ am Lehrstuhl für Abfallverwertungstechnik und Abfallwirtschaft (AVAW) der Montanuniversität Leoben

stellers für die Umweltauswirkungen eines Produktes auf den gesamten Lebenszyklus (Herstellung, Nutzung, Reparatur, Entsorgung, Behandlung und Rückführung als Sekundärrohstoff) aus. Zumeist sind damit Rücknahme- und Verwertungspflichten verbunden. EPR wird derzeit in der EU vor allem auf Verpackungen, Elektro- und Elektronikaltgeräte, Lampen, Batterien, Altfahrzeuge und Reifen angewendet. Das EU-Kreislaufwirtschaftspaket bietet die Chance, EPR zur Internalisierung externer Kosten und ökologisch sinnvoller Lenkungsanreize weiterzuentwickeln, insbesondere durch bindende Mindestanforderungen an Systeme der Produzentenverantwortung und Ausweitung auf neue Produktbereiche.

EPR verlagert die Verantwortung für Rücknahme und Verwertung auf Erzeuger und soll dadurch einen wirtschaftlichen Anreiz für Ökodesign, verbesserte Sammelsysteme und mehr Recycling schaffen. Schwachstellen liegen u.a. in fehlender Transparenz der Mengenströme und Verwertungsleistungen, Finanzierungsschwächen und in Aus- weichverhalten durch unzureichende Rechtsrahmen oder Vollzugsdefizite.

Es ist zu beachten, dass EPR-Modelle vielfach mit Vorauszahlungen arbeiten, die Kapital und Kaufkraft der Konsumenten binden. Die Eignung für Produkte oder Güter mit langer Nutzungsdauer ist daher im Einzelfall zu prüfen.

Die erweiterte Herstellerverantwortung liegt zwar im Bereich der Produzenten und Inverkehrbringer, muss jedoch auch auf regionaler Ebene eingefordert werden. Seit dem Kreislaufwirtschaftspaket 2018 ist die Herstellerverantwortung noch einmal präzisiert und in der SUP-Richtlinie 2019 auf den Bereich der gelitterten Abfälle ausgedehnt worden (ÖWAV 2018).

\section{Resümee}

Zukünftig sollten wir die Recyclingfähigkeit neu definieren. Erkennbarkeit und Sortierfähigkeit, aber auch die Eignung der Sammelsysteme sind unbedingt zu berücksichtigen. Die Prüfung der Recyclingfähigkeit muss auch durch experimentelle Tests abgesichert wer- den. Die Montanuniversität Leoben hat dazu in einen Versuchstand für sensorgestützte Sortierung investiert und wird in einem Technikum auch eine Versuchsanlage für Schüttgutanalyse aufbauen, um die praktische Recyclingfähigkeit untersuchen zu können.

Wenn wir zukünftig mehr und besser recyclingfähige Produkte in unseren abfallwirtschaftlichen Anlagen finden, werden wir technisch auch in der Lage sein, diese zu separieren und zu verwerten. Der Gesetzgeber ist daher aufgerufen für diese Privilegierung von recyclingfähigen Produkten die geeigneten Rahmenbedingungen und Anreizsysteme zu schaffen. Ökomodulation ist dazu ein wirksames Mittel.

Funding Open access funding provided by Montanuniversität Leoben.

Open Access Dieser Artikel wird unter der Creative Commons Namensnennung 4.0 International Lizenz veröffentlicht, welche die Nutzung, Vervielfältigung, Bearbeitung, Verbreitung und Wiedergabe in jeglichem Medium und Format erlaubt, sofern Sie den/die ursprünglichen Autor(en) und die Quelle ordnungsgemäß nennen, einen Link zur Creative Commons Lizenz beifügen und angeben, ob Änderungen vorgenommen wurden.

Die in diesem Artikel enthaltenen Bilder und sonstiges Drittmaterial unterliegen ebenfalls der genannten Creative Commons Lizenz, sofern sich aus der Abbildungslegende nichts anderes ergibt. Sofern das betreffende Material nicht unter der genannten Creative Commons Lizenz steht und die betreffende Handlung nicht nach gesetzlichen Vorschriften erlaubt ist, ist für die oben aufgeführten Weiterverwendungen des Materials die Einwilligung des jeweiligen Rechteinhabers einzuholen.

Weitere Details zur Lizenz entnehmen Sie bitte der Lizenzinformation auf http://creativecommons.org/licenses/ by/4.0/deed.de. 


\section{Literatur}

ECR Austria (2020): Packaging Design for Recycling.

cyclos-HTP (2019): Prüfung und Testierung de Recyclingfähigkeit - Anforderungs- und Bewertungskatalog des Institutes cyclos-http zur EUweiten Zertifizierung.

Europäische Kommission (2018): Eine europäische Strategie für Kunststoffe in der Kreislaufwirtschaft. Mitteilung der Kommission an das Europäische Parlament, den Rat, den Europäischen Wirtschafts-und Sozialausschuss und den Ausschuss der Regionen. COM(2018) 28 final. Europäisches Parlament (2019): Richtlinie (EU) 2019/904 des Europäischen Parlaments und des Rates vom 05. Juni 2019 über die Verringerung der Auswirkungen bestimmter Kunststoffprodukte auf die Umwelt.
Kronen Zeitung (2020): vom 14.08.2020

ÖWAV (2018): Strategien der österreichischen Recycling- und Abfallwirtschaft, Positionspapier des Österreichischen Wasser und Abfallwirtschafts Verbandes (ÖWAV).

Pomberger, R. (2019): 20 Jahre Abfallwirtschaft Wohin geht die Reise? Vortrag zum 20-Jahr-Jubiläum des V.EFB.

Stiftung Zentrale Stelle Verpackungsregister (2019): Mindeststandard für die Bemessung der Recyclingfähigkeit von systembeteiligungspflichtigen Verpackungen gemäß $§ 21$ Abs. 3 VerpackG.

Tacker, M., Krottendorfer, E. (2020): Circular Packaging Design, FH Campus Wien.

Wellacher, M., Schaffernak, A., Lichtenegger, S. (2020): Umsetzung der EU-Kunststoffstrategie in der Steiermark - Ausgangslage, Potenziale, technische Möglichkeiten und Maßnahmen. Studie im Auftrag vom Amt der Steiermärkischen Landesregierung, Montanuniversität Leoben (Hrsg.), Lehrstuhl für Abfallverwertungstechnik und Abfallwirtschaft, Leoben.

Hinweis des Verlags Der Verlag bleibt in Hinblick auf geografische Zuordnungen und Gebietsbezeichnungen in veröffentlichten Karten und Institutsadressen neutral. 